\title{
O MEDO, A ANSIEDADE E AS SUAS PERTURBAÇÕES
}

\author{
Américo Baptista ${ }^{1}$ \\ Marina Carvalho ${ }^{2}$ \\ Fátima Lory ${ }^{3}$
}

Resumo: É apresentado um modelo para compreensão do medo e da ansiedade, assim como de tratamento das suas perturbações, de acordo com o modo de actuação no Centro de Aconselhamento para Estudantes da Universidade Lusófona. O medo é considerado uma adaptação, de acordo com uma perspectiva evolutiva e desenvolvimentista. Como qualquer adaptação varia, podendo apresentar desajustamentos nos extremos da distribuição, tanto na baixa como na alta ansiedade, que designamos por perturbações hipoansiosas e hiperansiosas. São apresentadas as possibilidades de tratamento psicológico cognitivo-comportamental nas perturbações de hiperansiedade, assim como são discutidos os efeitos do tratamento, em diversos pârametros, e as possibilidades de prevenção.

Palavras-chave: medo, ansiedade, tratamento psicológico cognitivo-comportamental.

Fear, anxiety, and its disorders (abstract): In this paper we present a model for the understanding of fear and anxiety as well as the treatment of its disorders, in agreement with the methods used in the Centro de Aconselhamento para Estudantes da Universidade Lusófona. According to an evolutionary and developmental perspective, fear is viewed as an adaptation. As such, it varies and can show different maladjustments, both in high and low anxiety, that are normally designated as hypo and hyper-anxious disorders. We present possibilities of cognitive-behavioural psychological treatment of hyper-anxious disorders, as well as discuss its effects.

\footnotetext{
${ }^{1}$ Centro de Estudos de Psicologia Cognitiva e da Aprendizagem, Centro de Aconselhamento para Estudantes, Departamento de Psicologia da Universidade Lusófona. E-mail: americo.baptista@ulusofona.pt

2 Centro de Estudos de Psicologia Cognitiva e da Aprendizagem, Centro de Aconselhamento para Estudantes, Departamento de Psicologia da Universidade Lusófona.

${ }^{3}$ Centro de Estudos de Psicologia Cognitiva e da Aprendizagem, Centro de Aconselhamento para Estudantes, Departamento de Psicologia da Universidade Lusófona.
} 
Diversos rótulos verbais são utilizados para descrever um estado emocional desagradável de apreensão ou tensão, acompanhado por sintomas de activação fisiológica, como, por exemplo, palpitações, dificuldades em respirar, tonturas, suores, sensações de calor e frio ou tremores, desencadeados por uma ameça real ou antecipada (Baptista, 1988). Medo e ansiedade são os descritores mais utilizados, tanto na linguagem do dia-a-dia como na literatura psicológica. O termo angústia é cada vez menos utilizado, enquanto que, principalmente após a publicação da $3 .^{\text {a }}$ edição do manual de classificação e diagnóstico da Associação Psiquiátrica Americana (APA, 1980), o termo pânico tem vindo a ser utilizado cada vez mais frequentemente. Apesar de medo e ansiedade serem muitas vezes considerados sinónimos, a presença ou ausência de estímulos desencadeadores externos e o comportamento de evitação costumam ser as características que se utilizam para diferenciar os dois estados. Considera-se medo quando existe um estímulo desencadeador externo óbvio que provoca comportamento de fuga ou evitação, enquanto que ansiedade é o estado emocional aversivo sem desencadeadores claros que, obviamente, não podem ser evitados.

Do ponto de vista das teorias das emoções, o medo é considerado como uma emoção básica, fundamental, discreta, presente em todas as idades, culturas, raças ou espécies, enquanto que a ansiedade é uma mistura de emoções, na qual predomina o medo (Barlow, 2002; Ekman \& Davidson, 1994; Lewis \& Haviland Jones, 2000; Plutchik, 2003). Como mistura de emoções, a fenomenologia da ansiedade é mais variável que a do medo. Pode variar ao longo do tempo ou de acordo com as situações desencadeadoras, sendo, assim, mais vaga, imprecisa e difícil de definir. Quando é um modo habitual e consistente de reacção designa-se por "ansiedade traço", quando é uma reacção episódica ou situacional designa-se por "ansiedade estado" (Spielberger, 1985). A ansiedade pode incluir a tristeza, a vergonha e a culpa, como pode, igualmente, ser composta por cólera, curiosidade, interesse ou excitação.

A ansiedade e o medo, apesar das questões semânticas a propósito da sua distinção, podem, ainda, ser diferenciados pela reacção na eventual presença de um potencial predador, identificado pelo odor, ou pela presença real de um predador, detectado pela visão (Gray \& McNaugton, 2000). Também as estruturas neurológicas que lhes servem de suporte parecem ser diferentes. Os percursos cerebrais implicados no medo e na ansiedade em humanos não estão completamente estabelecidos, mas a investigação animal aponta para sistemas, tanto anatómica como farmacologicamente, diferentes (Davis, 1997; Damásio, Adolphs \& Damásio, 2003).

Apesar das diversas diferenças entre medo e ansiedade, a teoria bio-informacional das emoções apresenta uma perspectiva integradora de ambas (Bradley \& Lang, 2000; Lang, 1978, 1993). As emoções são conside- 
radas como tendências ou disposições para a acção e é assumida uma estrutura motivacional que varia da aproximação à fuga ou do apetite à defesa. As emoções associadas ao sistema apetitivo relacionam-se com comportamentos de alimentação, sexualidade e carinho, enquanto que as emoções típicas do sistema defensivo se relacionam com comportamentos de protecção e defesa, como o vómito, a fuga, a evitação ou a reaç̧ão de congelamento. Qualquer emoção, de acordo com este modelo, pode ser definida ou medida em três grandes sistemas de resposta: os comportamentos observáveis ou as sequências comportamentais funcionais, a linguagem emocional ou a comunicação expressiva e as reacções fisiológicas ou a preparação para essas respostas. Apesar desta organização, em três sistemas de respostas, ser uma simplificação do comportamento emocional, permite avaliar o medo e a ansiedade de acordo com a intensidade da expressão de cada uma das dimensões. A dimensão comportamental, pela presença e intensidade de comportamentos de congelamento, evitação ou fuga; a dimensão subjectivo-verbal, pelo relato avaliativo do perigo ou ameaça implicados na situação; e a dimensão fisiológica, avaliada por indicadores de activação autonómica periférica, modificação de padrões de configuração muscular, alterações nos parâmetros dos sistemas imunitários e endocrinológicos e índices centrais no funcionamento do sistema nervoso. O medo ou a ansiedade podem, assim, ser definidos por uma constelação de medidas em cada um dos três sistemas de resposta: o que se faz, o comportamento, o que se pensa ou diz, a linguagem e o que se sente perante uma ameaça real ou imaginada, a fisiologia.

\section{Evolução e Desenvolvimento dos Medos}

Como parte do sistema defensivo, o medo e a ansiedade são activados por situações potencialmente ameaçadoras ou por perigos reais. Contudo, a compreensão destes aspectos implica, para além dos modelos gerais do comportamento emocional, uma perspectiva, simultaneamente, evolutiva ou filogenética e desenvolvimental ou ontogenética (Baptista, 2000; no prelo). A perspectiva evolutiva tem como objectivo a compreensão das causas últimas para o desencadeamento dos diversos tipos de medo, assim como os seus padrões automáticos de resposta. Por exemplo, as situações actualmente mais temidas, como os animais, cobras e aranhas, ou os locais e os ambientes mais temidos, como os espaços fechados ou muito amplos, sem abrigo, e as tempestades naturais, foram ameaças ancestrais, mas, hoje, são praticamente inofensivas devido às mudanças que a cultura introduziu no ambiente. No entanto, para os grupos nómadas de caçadores-recolectores, em que a espécie humana evoluiu e passou 
quase toda a sua existência, estas eram as ameaças reais. Hoje, não representam qualquer perigo, mas continuam a ser temidas. A vida humana dependeu do desenvolvimento de estratégias adequadas para lidar com essas ameaças. Seligman, (1971) foi, talvez, o primeiro investigador a chamar a atenção para este fenómeno, mostrando que o que é aparentemente irracional no medo é, apenas, uma vulnerabilidade para o aparecimento de "medos evolutivamente preparados", isto é, para temer as situações que, ao longo de milhões de anos de evolução, constituíram ameaças graves ou letais. Ao contrário, as ameaças reais actuais, sem nenhum significado evolutivo, dificilmente provocam medos (Seligman \& Hager, 1972). São raras as fobias relacionadas com electricidade, é inexistente o medo de ingerir alimentos calóricos ou de conduzir a alta velocidade e temos mais medo de dinossáurios que de armas de fogo.

Um outro aspecto, relevante do ponto de vista evolutivo, são os padrões automáticos de resposta. Perante um estímulo ameaçador, várias respostas são possíveis, como a evitação ou fuga, a imobilidade, a defesa agressiva ou a submissão. Mas a resposta não é aleatória, nem pensada, apesar de ter uma adequação perfeita ao tipo de ameaça (Baptista, 2000). Perante um predador, a resposta é de imobilidade ou fuga, perante um indivíduo ameaçador, a resposta é de submissão, como corar e, perante uma ameaça ambiental, como um declive abrupto, desencadeia-se uma reacção de imobilidade. Apesar da naturalidade e automacidade das respostas, a computação é enorme e a decisão é praticamente instantânea. Sobreviveram os que, ao longo da história evolutiva, não se enganaram, isto é, os que não coraram perante um predador, mas ficaram imóveis e não foram detectados, ou os que conseguiram escapar rapidamente e com sucesso.

Por outro lado, os medos, os distintos tipos de ansiedade e as suas desregulações aparecem num indivíduo em desenvolvimento, habitualmente, durante a infância até ao início da idade adulta, tendendo depois a diminuir com a idade (Essau \& Petermann, 2001; Last, 1993; March, 1995; Marks, 1987). Os medos aparecem e desaparecem, de modo previsível, em determinadas alturas do desenvolvimento, de acordo com as tarefas típicas dessas etapas, o que implica, para além de uma perspectiva evolutiva, uma perspectiva desenvolvimentista. Por exemplo, o medo das alturas tem início na altura do aparecimento da locomoção, por volta dos seis meses, e a sua intensidade correlaciona-se positivamente com a maestria nesta capacidade de explorar o ambiente. Apesar das limitações que este medo impõe, em associação com a ansiedade de separação, é um factor protector das ameaças apresentadas pelos declives ou pelo afastamento dos progenitores na fase inicial da vida. O estudo longitudinal de Dunedin, que seguiu crianças dos 3 aos 18 anos, mostrou que níveis elevados de medo na infância foram preditores de menos quedas e ferimentos durante a infância e a 
adolescência e que, ao contrário, a ausência de medo foi um factor de risco para acidentes pessoais (Menzies \& Parker, 2001; Poulton \& Menzies, 2002). Os medos reflectem a maturação do organismo, de acordo com as etapas do desenvolvimento. Igualmente, as suas manifestações variam ao longo da vida (Vasey \& Dadds, 2001). O choro e as birras podem ser, na infância, manifestações de medo que, gradualmente, são substituídos por outros sinais comportamentais expressivos. O medo deve ser considerado como um programa genético, aberto às influências do ambiente que, por processos de habituação ou de sensibilização, os podem fazer diminuir ou aumentar. Por exemplo, os medos sociais aparecem no início da adolescência, altura de maior individualização, de afastamento dos progenitores, de inserção nos grupos de pares e de atracção pelo sexo oposto. A preocupação com a aparência, o cuidado com a impressão causada e a opinião dos outros é, nesta altura, muito importante. O medo ou ansiedade social são factores motivadores adaptativos que promovem maior preocupação e cuidado com estes aspectos. Contudo, se este medo inibir os contactos sociais ou se existir encorajamento familiar para a evitação, o medo terá tendência a manter-se ou, pior, a aumentar transformando-se numa limitação.

\section{O Medo, a Ansiedade e as suas Perturbações}

O medo ou a ansiedade, como qualquer outra característica, variam. Um conjunto de indivíduos tem baixos níveis de ansiedade, outro conjunto tem altos níveis de ansiedade e entre estes dois extremos situam-se a maioria dos indivíduos com níveis intermédios de ansiedade. Utilizando como exemplo a ansiedade social, verifica-se que um conjunto reduzido de indivíduos é extremamente descontraído ou corajoso em situações novas ou sociais. No outro extremo da distribuição, situam-se os indivíduos que têm níveis de ansiedade social tão alta que evitam, sempre que possível, as situações sociais ou que apenas as enfrentam com um sofrimento enorme. Entre estes dois extremos situa-se a maioria das pessoas com níveis diversos de ansiedade social, mas que não interferem com o desempenho social. O mesmo acontece com os outros tipos de medo ou ansiedade.

O extremo da distribuição, constituído por pessoas com níveis baixos de ansiedade, devem ser considerados como apresentando uma perturbação de hipoansiedade. Este conceito foi introduzido por Marks e Nesse (1994) para descrever os indivíduos com uma resposta baixa e deficiente em relação a estímulos realmente ameaçadores que não os inibe de se entregarem a comportamentos de risco. As perturbações de externalização, como a perturbação de hiperactividade e défice da atenção, a perturbação da conduta, a perturbação desafiante por oposição, com início na infância ou 
adolescência, e a perturbação anti-social da personalidade da idade adulta, deverão estar relacionadas com limiares elevados de activação do sistema defensivo ou, eventualmente, com baixos limiares para activação do sistema apetitivo, como, por exemplo, nas perturbações relacionadas com o uso ou abuso de substâncias. Ao contrário, o outro extremo da distribuição é composto por indivíduos com perturbações da internalização, isto é, com baixos limiares para activação do sistema defensivo, como a perturbação por ansiedade de separação e o mutismo selectivo, com início na infância ou adolescência, ou as perturbações ansiosas da idade adulta, como a perturbação de pânico com ou sem agorafobia, a fobia específica, a fobia social ou perturbação de ansiedade social, a perturbação obsessivo-compulsiva, a perturbação pós-stress traumático, a perturbação aguda ao stress e a perturbação de ansiedade generalizada (APA, 2000; WHO, 1992).

\section{Possibilidades de Tratamento}

Apesar das perturbações do medo e da ansiedade terem sido consideradas como os dois extremos da distribuição, isto é, tanto de baixa como de alta ansiedade, não existe, actualmente, nenhum modelo integrador para o tratamento de ambas. Para as perturbações de alta ansiedade, os tratamentos farmacológicos e os tratamentos psicológicos mostraram ser particularmente eficazes, sendo conhecidos para cada um deles as suas indicações, vantagens e limitações (Barlow, 2002).

Apesar da sua diferencial aplicabilidade, através das diferentes perturbações de ansiedade, os tratamentos farmacológicos com eficácia demonstrada são as benzodiazepinas, os antidepressivos tricíclicos e os inibidores da recaptação da serotonina. Por exemplo, nas fobias específicas, estes tratamentos são de utilidade reduzida, enquanto que nas perturbações ansiosas mais graves, como a perturbação de ansiedade social, a perturbação de pânico ou a perturbação obsessivo-compulsiva, são particularmente eficazes, principalmente nas fases agudas ou quando existe co-morbilidade com as perturbações do humor.

Os tratamentos psicológicos que demonstraram ser eficazes na diminuição da ansiedade baseiam-se em dois tipos de procedimentos, empiricamente suportados, que se designam por terapia comportamental e por terapia cognitiva (Baptista, 1999). A terapia comportamental tem como objectivos a modificação dos comportamentos relacionados com o medo, a evitação ou a fuga, e a diminuição da activação fisiológica. A terapia cognitiva tem como objectivos a alteração dos conteúdos e dos processos cognitivos relacionados com o medo, as interpretações catastróficas e a atenção. A aplicação integrada dos dois procedimentos, tal como é habi- 
tualmente feito, designa-se por psicoterapia cognitivo-comportamental. Um dos aspectos mais interessantes destas intervenções é a possível utilização diferencial dos dois percursos cerebrais do medo, identificados por LeDoux (1998; 2002), pelos dois componentes do tratamento psicológico, a terapia comportamental pelo percurso subcortical e a terapia cognitiva pelo percurso cortical (Baptista, no prelo, a).

A terapia cognitivo-comportamental para as perturbações da ansiedade encontra-se actualmente descrita em diversos manuais para cada uma das perturbações ansiosas, tanto para crianças como para adultos (Andrews, Creamer, Crino, Hunt, Lampe \& Page 2003; Carr, 2000; Christopherson \& Mortweet, 2001; Kasdin \& Weisz, 2003; Nathan \& Gorman, 1998; Reinecke, Dattilio \& Freeman, 2003; Roth \& Fonagy, 1996; Silverman \& Treffers, 2001). De um modo geral, e independentemente das características de cada uma das perturbações, o modelo de intervenção é composto por três fases. Uma fase educativa, onde é dada informação a propósito dos factores relacionados com a génese, manutenção e tratamento, em que é enfatizada a necessidade de colaboração do paciente, assim como o possível envolvimento de familiares ou pessoas significativas do seu ambiente social. Nesta fase, é iniciado um procedimento de auto-observação ou automonitorização em que se estabelecem os alvos do tratamento, assim como os factores individuais relacionados com a manutenção do comportamento e emoções perturbadoras. Na segunda fase, são ensinadas aptidões que, no caso das perturbações da ansiedade, têm como finalidade eliminar os comportamentos de fuga, evitação ou segurança, provocar a diminuição das respostas ansiosas, nomeadamente, a activação fisiológica e a alteração das crenças ou dos padrões disfuncionais de pensamento. Após o domínio destas aptidões, é efectuado, na terceira fase, o confronto, de modo sistemático, com as situações ansiogénicas para diminuir as respostas ansiosas e aumentar as expectativas de auto-eficácia. No caso das crianças e dos adolescentes, estes protocolos de tratamento são adaptados ao seu estádio desenvolvimental, assim como é efectuado um maior envolvimento no tratamento por parte da família e de outros agentes de socialização (Baptista, Pereira, Carvalho, Lory \& Santos, 2001).

\section{Conclusões}

$\mathrm{O}$ medo e a ansiedade devem ser considerados como alarmes ou adaptações avisadoras dos perigos que a espécie humana enfrentou ao longo da sua história evolutiva. São, igualmente, adaptações afinadas para as previsíveis tarefas ao longo da história da vida, assim como de acordo com o grau de ameaça ambiental. Os medos aparecem quando são úteis, 
como a ansiedade de separação, o medo das alturas ou a ansiedade social, e desaparecem, habitualmente, quando essas tarefas estão suficientemente dominadas e deixam de constituir ameaça. A aplicação da teoria da evolução pela selecção natural tem sido frequentemente criticada por criar histórias ou modelos interpretativos que não podem ser cientificamente testados. Contudo, a acumulação de conhecimentos tem confirmado a maioria das hipóteses que têm sido formuladas (Õhman, 2000; Õhman \& Wiens, 2003). Um estudo recente efectuado na Noruega mostrou como os predadores carnívoros, ursos e lobos, continuam a ser percebidos como ameaçadores, de acordo com os papéis sexuais do passado evolutivo da espécie humana (Roskaft, Bjerke, Kaltenborn, Limmell \& Anderson, 2003).

Apesar das contribuições filogenéticas, o medo e a ansiedade são programas modificáveis por factores ambientais que os podem aumentar ou diminuir, que os podem fazer aparecer, como nas situações traumáticas, ou que os podem manter para além dos períodos normalmente esperados. Por exemplo, a protecção parental ou limitações no comportamento exploratório podem retardar a diminuição dos medos normais ou mantê-los para além do período esperado, transformando-os em fobias; ou um acontecimento traumático como, por exemplo, o 11 de Setembro, pode duplicar o número de perturbações ansiosas em quem está fisicamente próximo do acontecimento (Galea, Ahern, Resnick, Kilpatrick, Bucuvalas, Gold, \& Vlahov, 2002).

$\mathrm{O}$ medo e a ansiedade variam, tanto no limiar com que são activados, como na intensidade da resposta. Os indivíduos que necessitam de um grau de ameaça elevado ou em que a intensidade da resposta é baixa, assim como os que facilmente são activados e respondem de modo intenso, apresentam perturbações ou desregulações da resposta ansiosa. O primeiro grupo raramente é considerado como tendo uma perturbação da ansiedade, mas deve designar-se por hipofóbico ou hipoansioso, enquanto que o segundo, as perturbações tradicionais da resposta ansiosa, deve designar-se por hiperfóbico ou hiperansioso.

Os tratamentos actualmente à disposição para as perturbações de baixa ansiedade não são particularmente eficazes, ao contrário dos tratamentos para as perturbações relacionadas com altos níveis de ansiedade. Os tratamentos cognitivo-comportamentais para as perturbações fóbicas e ansiosas são dos maiores sucessos da história da psicoterapia, comparando-se favoravelmente com qualquer outro tratamento actualmente à disposição, quando se analisam quer resultados de eficácia clínica, quer outros parâmetros como as relações de custo/eficácia, as taxas de recaídas, a aceitabilidade ou os efeitos secundários do tratamento (Baptista, no prelo; Baptista, Pereira, Lory, Rosa, Dias, Carvalho \& Gonzalez, 2003; Barlow, 2002; Otto, Pollack, \& Maki, 2000). A conceptualização das perturbações 
do medo ou da ansiedade como podendo existir nos dois extremos da distribuição, isto é, tanto de baixa como de alta ansiedade, assim como a compreensão destas emoções, de um ponto de vista evolutivo e desenvolvimentista, pode ajudar a abrir novas perspectivas tanto para a prevenção como para o tratamento das suas perturbações.

\section{Referências}

American Psychiatric Association. (1980). Diagnostic and statistical manual of mental disorders (3rd ed.). Washington, DC: Author.

American Psychiatric Association. (2000). Diagnostic and statistical manual of mental disorders (4th ed., Text revision). Washington, DC: Author.

Andrews, G., Creamer, M., Crino, R., Hunt, C., Lampe, L., \& Page, A. (2003). The treatment of anxiety disorders. Clinician guides and patient manuals (2nd Ed.). Cambridge: Cambridge University Press.

Baptista, A. (1988). Ansiedade I. Natureza e classificação. Revista de Psiquiatria do Hospital Júlio de Matos, 1, 149-164.

Baptista, A. (1999). Eficácia e disseminação dos programas psicológicos de tratamento. O desafio actual. Revista de Humanidades e Tecnologias, 1, 52-61.

Baptista, A. (2000). Perturbações do medo e da ansiedade. Uma perspectiva evolutiva e desenvolvimental. In I. Soares (Ed.), Psicopatologia do desenvolvimento. Trajectórias (in)adaptativas ao longo da vida. Coimbra: Quarteto.

Baptista, A. (no prelo). Teoria de la selección natural, psicología evolucionista y emociones. Ansiedad y Estrés.

Baptista, A. (no prelo, a). Intervenções psicológicas para o tratamento e prevenção da perturbação de pânico. Porto: Bial.

Baptista, A. Pereira, A. C., Carvalho, M., Lory, F., \& Santos, R. (2001). Aprender a lidar com as dificuldades emocionais. O centro de aconselhamento para estudantes. Revista de Humanidades e Tecnologias, 4/5, 227-237.

Baptista, A., Pereira, A. C., Lory, F., Rosa, J. B., Dias, M., Carvalho, M., \& Gonzalez, B. (2003). Centro de Aconselhamento para Estudantes. Relatório de actividades de Setembro de 2002 a Julho de 2003. Boletim de Psicologia da Universidade Lusófona, 8, 10-14.

Barlow, D. H. (2002). Anxiety and its disorders. The nature and treatment of anxiety and panic (2nd ed.). Nova Iorque: Guilford.

Bradley, M., \& Lang, P. (2000). Measuring emotion: Behavior, feeling, and physiology. In R. D. Lane \& L. Nadel (Eds.), Cognitive neuroscience of emotion (pp. 242-276). Oxford: Oxford University Press.

Carr, A. (2000). What works with children and adolescents? A critical review of psychological interventions with children, adolescents and their families. Londres: Routledge. 
Christopherson, E. R., \& Mortweet, S. L. (2001). Treatments that work with children. Empirically supported strategies for managing childhood problems. Washington, DC: American Psychological Association.

Damásio, A. R., Adolphs, R., \& Damásio, H. (2003). The contributions of the lesion method to the functional neuroanatomy of emotion. In R. J. Davidson, K. S. Scherer, \& H. H. Goldsmith (Eds.), Handbook of affective sciences (pp. 66-87). Oxford: Oxford University Press.

Davis, M. (1997). Neurobiology of fear responses: The role of the amygdala. Journal of Neuropsychiatry and Clinical Neurosciences, 9, 382-402.

Ekman, P., \& Davidson, R. J. (1994). The nature of emotion. Fundamental questions. Oxford: Oxford University Press.

Essau, C., \& Petermann, F. (2001). Anxiety disorders in children and adolescents. Epidemiology, risk factors and treatment. Hove: Taylor and Francis.

Galea, S., Ahern, J., Resnick, H., Kilpatrick, D., Bucuvalas, M., Gold, J., \& Vlahov, D. (2002). Psychological sequelae of the September 11 terrorists attacks in New York City. New England Journal of Medicine, 346, 982-987.

Gray, J., \& McNaugton, N. (2000). The neuropsychology of anxiety (2nd Ed.). Oxford: Oxford University Press.

Kazdin, A. E., \& Weitsz, J. R. (2003). Evidence-based psychotherapies for children and adolescents. Nova Iorque: Guilford.

Lang, P. (1979). A bio-informational theory of emotional imagery. Psychophysiology, 16, 495-512.

Lang, P. (1993). The three system approach to emotion. In N. Birbaumer \& A. Õhman (Eds), The organization of emotion (pp. 18-30). Toronto: Hogrefe-Huber.

Last, C. G. (1993). Anxiety across the life-span: A developmental perspective. Nova Iorque: Springer.

LeDoux, J. (1998). The emotional brain. The mysterious underpinnings of emotional life. Londres: Weindenfeld \& Nicolson.

LeDoux, J. (2002). Synaptic self. How our brains became who we are. Nova Iorque: Macmillan.

Lewis, J., \& Haviland Jones, J. M. (2000). Handbook of emotions (2nd Ed.). Nova Iorque: Guilford Press.

March, J. S. (1995). Anxiety disorders in children and adolescents. Nova Iorque: Guilford Press.

Marks, I. M. (1987). Fears, phobias and rituals. Panic, anxiety and their disorders. Oxford: Oxford University Press.

Marks, I. M., \& Nesse, R. M. (1994). Fear and fitness. Ethology and Sociobiology, $15,247-261$.

Menzies, R. G., \& Parker, L. (2001). The origins of height fear: An evaluation of conditioning explanations. Behaviour Research and Therapy, 39, 185-199.

Nathan, P. E., \& Gorman, J. M. (1998). A guide to treatments that work. Oxford: Oxford University Press. 
Õhman, A. (2000). Fear and anxiety: Evolutionary, cognitive and clinical perspectives. In M. Lewis \& J. M. Haviland-Jones (Eds.), Handbook of emotions (2nd Ed.). Nova Iorque: Guilford Press.

Õhman, A., \& Wiens, R. (2003). On the automaticity of autonomic responses in emotion: An evolutionary perspective. In R. J. Davidson, K. S. Scherer \& H. H. Goldsmith (Eds.), Handbook of affective sciences (pp. ?? -??). Oxford: Oxford University Press.

Otto, M. W., Pollack, M. H., \& Maki, K. M. (2000). Empirically supported treaments for panic disorder: Costs, benefits and stepped care. Journal of Consulting and Clinical Psychology, 68, 556-563.

Plutchik, R. (2003). Emotions and life. Perspectives from psychology, biology, and evolution. Washington, DC: American Psychological Association.

Poulton, R., \& Menzies, R. G. (2002). Non-associative fear acquisition: A review of the evidence from retrospective and longitudinal research. Behaviour Research $<$ and Therapy, 40, 127-149.

Reinecke, M. A., Dattilio, F. M., \& Freeman, A. (2003). Cognitive therapy with children and adolescents. Nova Iorque: Guilford.

Roskaft, E., Bjerke, T., Kaltenborn, B., Limmell, J. D. C., \& Anderson, R. (2003). Patterns of self-reported fear toward large carnivores among the Norwegian public. Evolution and Human Behavior, 24, 184-198.

Roth, A., \& Fonagy, P. (1996). What works for whom? A critical review of psychotherapy research. New York: The Guilford Press.

Seligman, M. (1971). Phobias and preparedness. Behavior Therapy, 2, 307-320.

Seligman, M. \& Hager, J. L. (1972). Biological boundaries of learning. Nova Iorque: Appleton Century Crofts.

Silverman, W. K., \& Treffes, P. D. A. (2001). Anxiety disorders in children and adolescents. Research, assessment and intervention. Cambridge: Cambridge University Press.

Spielberger, C. D. (1985). Anxiety, cognition and affect: A state-trait perspective. In A. Tuma \& J. D. Maser (Eds.), Anxiety and the anxiety disorders (pp ?? -??). Hillsdale, NJ: Earlbaum.

Vasey, M. W., \& Dadds, M. R. (2001). The developmental psychopathology of anxiety. Oxford: Oxford University Press.

World Health Organization. (1992). International classification of diseases, 10th revision (ICD-10). Geneva: World Health Organization. 